\title{
Pericarditis tuberculosa en paciente inmunocompetente. Reporte de caso
}

\section{Tuberculous pericarditis in an immunocompetent patient. Case report}

\author{
Daniel Felipe Reyes-Vega (iD) ${ }^{1}$, Kenny Buitrago-Toro (iD) ${ }^{2}$, Silvana Jiménez-Salazar ${ }^{(\mathbb{D})^{3}}$, Luis Carlos-Álvarez (iD) 4 \\ 1. Hospital Universitario Hernando Moncaleano Perdomo. Neiva, Colombia. Correo:_danfereve@ hotmail.com - https://orcid.org/0000-0002-7873-0121 \\ 2. Universidad Surcolombiana. Neiva, Colombia. Correo: kenny9012@hotmail.com - https://orcid.org/0000-0001-8145-5194 \\ 3. Universidad Surcolombiana. Neiva, Colombia. Correo: silvana1212@gmail.com - https://orcid.org/0000-0003-3045-1331 \\ 4. Universidad de Antioquia. Medellín, Colombia. Correo: kapomed@Live.com - https://orcid.org/0000-0001-8227-810X
}

Tipología: Reporte de caso clinico

Para citar este artículo: Reyes-Vega DF, Toro-Buitrago K, Jiménez-Salazar S, Álvarez LC. Pericarditis tuberculosa en paciente inmunocompetente. Reporte de caso. Duazary. 2021 enero;18(1): 99-106. Doi: https://doi.org/10.21676/2389783X.3834

Recibido en noviembre 07 de 2019

Aceptado en noviembre 11 de 2020

Publicado en línea en enero 07de 2021

Palabras

clave:

pericarditis; tuberculosis; adenosina desaminasa.

\section{RESUMEN}

La pericarditis tuberculosa es una rara manifestación extrapulmonar que afecta a menos del $4 \%$ de los pacientes con tuberculosis (TB), con alta morbimortalidad ante diagnósticos tardíos, especialmente en aquellos pacientes inmunocomprometidos. Su presentación es inespecífica y la sintomatología deriva de la velocidad de instauración del derrame pericárdico, registrándose casos mortales debido a taponamiento cardiaco. La confirmación diagnóstica se obtiene con la identificación de la micobacteria en muestra de pericardio o líquido pericárdico, con una alta tasa de sospecha fundamentada en pruebas adicionales como la cuantificación del interferón gamma no estimulado, lisozima pericárdica y adenosina desaminasa (ADA) en líquido pericárdico. El tratamiento consiste en una combinación de terapia antituberculosa, drenaje pericárdico y corticoesteroides como adyuvantes. Se presenta el caso de un varón de 64 años inmunocompetente, fumador frecuente en el pasado, con una alta sospecha diagnóstica para TB, confirmada con resultado positivo para ADA $(55,8 \mathrm{UI} / \mathrm{L})$ en fluido pericárdico. Esto permitió iniciar un tratamiento adecuado con mejoría clínica posterior durante el seguimiento.

\section{ABSTRACT}

Keywords: Pericarditis; Tuberculosis; Adenosine Deaminase.

Tuberculous pericarditis is a rare extrapulmonary manifestation that affects less than $4 \%$ of patients with tuberculosis (TB). It has high morbidity and mortality at late diagnoses, especially in immunocompromised patients. Its presentation is unspecific. The symptomatology derives from the speed of installation of the pericardial effusion, with fatal cases being recorded due to cardiac tamponade. Diagnostic confirmation is obtained with the identification of mycobacteria in a pericardial sample fluid, with a high suspicion rate based on additional tests such as quantification of non-stimulated gamma interferon, pericardial lysozyme, and adenosine deaminase (ADA) in pericardial fluid. The treatment consists of a combination of anti-tuberculous therapy, pericardial drainage, and corticosteroids as an adjuvant. We present the case of a 64-year-old immunocompetent male, a frequent smoker in the past, with a high diagnostic suspicion for TB, confirmed positive for ADA (55,8 UI/L) in pericardial fluid. That allowed initiating an adequate treatment with subsequent clinical improvement during the follow-up. 


\section{INTRODUCCIÓN}

La pericarditis tuberculosa es una manifestación extrapulmonar infrecuente de la tuberculosis (TB), con una incidencia de presentación menor al $4 \%$ de todos los casos de la enfermedad ${ }^{1}$. Se instaura por diseminación linfática desde los ganglios peritraqueales, peribronquiales y mediastinales, por diseminación hematógena o por vecindad con tuberculosis pleural o pulmonar ${ }^{1,2}$. Su frecuencia aumenta en áreas endémicas, en pacientes ancianos o con inmunodeficiencia de base $e^{3-5}$. Esta condición tiene una presentación clínica variable y poco específica ${ }^{2}$, con una mortalidad de 80 a $90 \%$ si no se trata adecuadamente, especialmente cuando el diagnóstico es tardío y retrasa el tratamiento ${ }^{6}$.

El diagnóstico se fundamenta en la sospecha clínica, sumado a los hallazgos imagenológicos en radiografía, tomografía computarizada (TC) de tórax y ecocardiograma, y se confirma con el aislamiento de la micobacteria a través de cultivo, biopsia o reacción en cadena de la polimerasa $(P C R)^{1-4}$. Estudios adicionales del líquido pericárdico como la cuantificación del interferón gamma no estimulado, la ADA y la lisozima pericárdica, son especialmente útiles en aquellos casos en los cuales no es posible aislar el microorganismo, facilitando el inicio temprano de la terapia antibiótica ${ }^{6}$.

Se presenta el caso de un varón de 64 años, fumador frecuente, inmunocompetente, con diagnóstico de pericarditis tuberculosa confirmada mediante cuantificación de ADA en líquido pericárdico, a quien se realiza tratamiento oportuno con una adecuada recuperación y alta a los 20 días de estancia hospitalaria.

\section{REPORTE DE CASO CLÍNICO}

Paciente masculino de 64 años de edad, inmunocompetente, con antecedentes de tabaquismo frecuente, Índice Paquete Año (IPA) de 10, suspendido hace 10 años, sin nexos epidemiológicos de exposición infecciosa ni antecedentes patológicos, quien consulta inicialmente a un hospital de mediana complejidad refiriendo cuadro clínico de 3 días de evolución consistente en fiebre subjetiva asociada a escalofríos, dolor pleurítico de predominio izquierdo, exacerbado a la palpación de la pared costal, además de tos, ortopnea y disnea de pequeños esfuerzos. Le realizan TC de tórax y encuentran un extenso derrame pericárdico y pleural izquierdo, por lo que derivan a nuestra institución para realización de drenaje pericárdico. Al ingreso se encuentra el paciente con signos vitales estables TA: 120/75 mmHg, Fc: 93 Ipm, Fr: 17 rpm SO2: $96 \%$ y $\mathrm{T}: 36,8{ }^{\circ} \mathrm{C}$, sin disnea, con persistencia de dolor en hemitórax izquierdo. Al examen físico hay presencia de ingurgitación yugular grado I, ruidos cardiacos arrítmicos y dolor a la palpación profunda en hipocondrio izquierdo.

Se realizó hemograma que evidenció leucocitosis de $17.000 / \mathrm{mm}^{3}$ con neutrofilia del $85 \%$, glucemia: 98,9 $\mathrm{mg} / \mathrm{dL}$ y positividad de marcadores inflamatorios, VSG: $65 \mathrm{~mm} / \mathrm{h}, \quad$ PCR: $34,58 \mathrm{mg} / \mathrm{L}$. El electrocardiograma mostró un ritmo de fibrilación de temporalidad indeterminada, no colapsante, sin alteraciones del segmento ST. Se realizó una ecografía de espacio pericárdico (Figura 1) en la que se reportó derrame pericárdico de aproximadamente $400 \mathrm{~mL}$ con excursión diafragmática bilateral preservada, y una radiografía de tórax (Figura 2) que evidenció cardiomegalia global y obliteración del ángulo costofrénico izquierdo por derrame pleural. La TC de tórax (Figura 3) confirmó la presencia de derrame pleural y pericárdico. Adicionalmente, el ecocardiograma transtorácico reportó hipertrofia cardiaca concéntrica no obstructiva de grado leve y signos indirectos de aumento de la presión intrapericárdica. Con base en los hallazgos clínicos y paraclínicos se estableció como diagnóstico inicial derrame pericárdico severo agudo de etiología a determinar. 

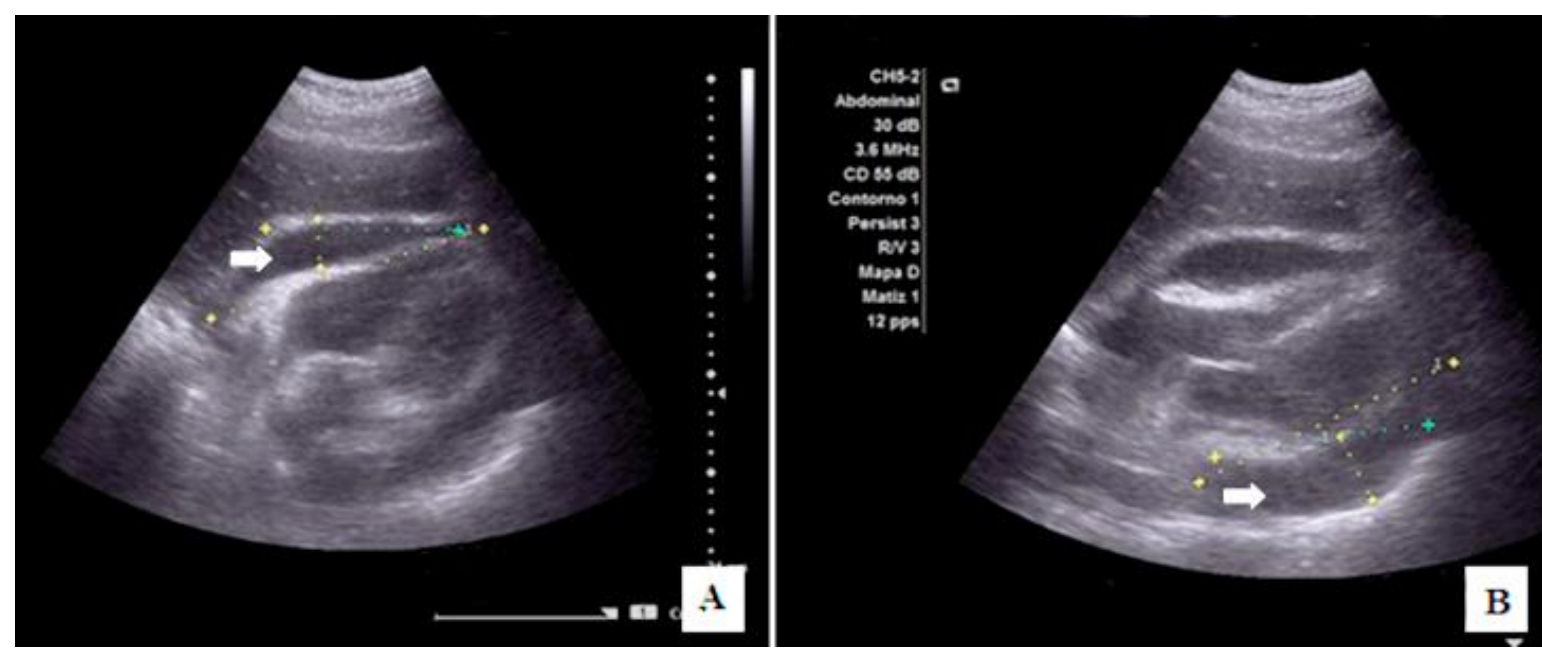

Figura 1. Ecografía de saco pericárdico, A y B: Imágenes en modo B, plano axial subxifoideo que muestra derrame pericárdico (flechas) con volumen aproximado de $400 \mathrm{~mL}$.

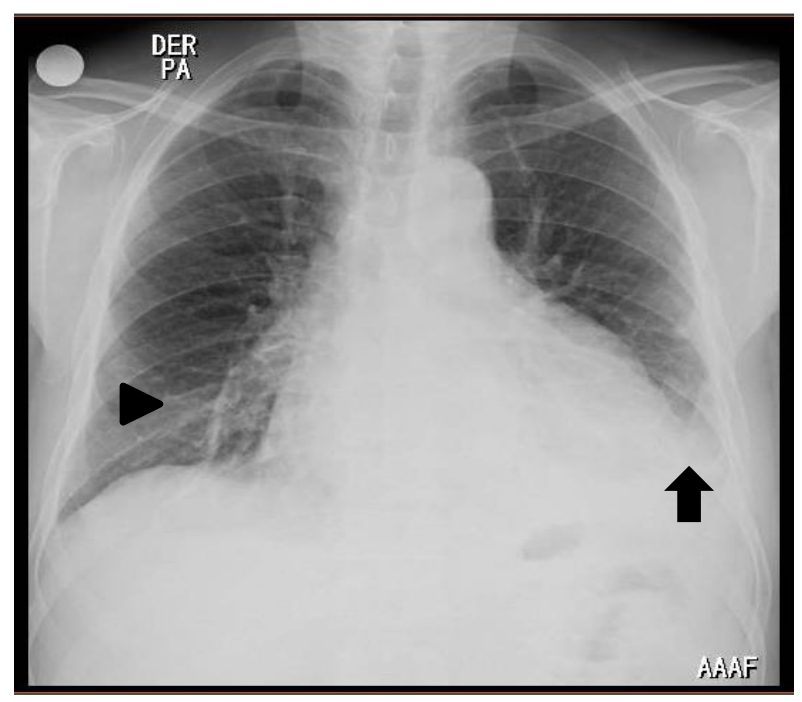

Figura 2. Radiografía de Tórax, proyección anteroposterior. Cardiomegalia global. Se observan bandas parenquimatosas bibasales atribuidas a atelectasia (punta de flecha) y obliteración del ángulo costodiafragmático izquierdo por derrame pleural (flecha).

Se realizó drenaje pericárdico y se extrajeron aproximadamente $700 \mathrm{~mL}$ de líquido seroso de apariencia turbia. Se tomó muestra para cultivo de micobacterias y coloración de Ziehl-Neelsen los cuales fueron negativos para bacilos acido-alcohol resistentes. Los resultados del estudio citoquímico se muestran en detalle en la Tabla 1 . Se realizó análisis de ADA en líquido pericárdico, el cual fue positivo con valores de 55,8 UI/L, y se obtuvo biopsia de pericardio en la que se evidenció presencia del bacilo de Koch e inflamación crónica, sin presencia de proceso neoplásico o granulomatoso. Esto permitió establecer la etiología del derrame pericárdico. 


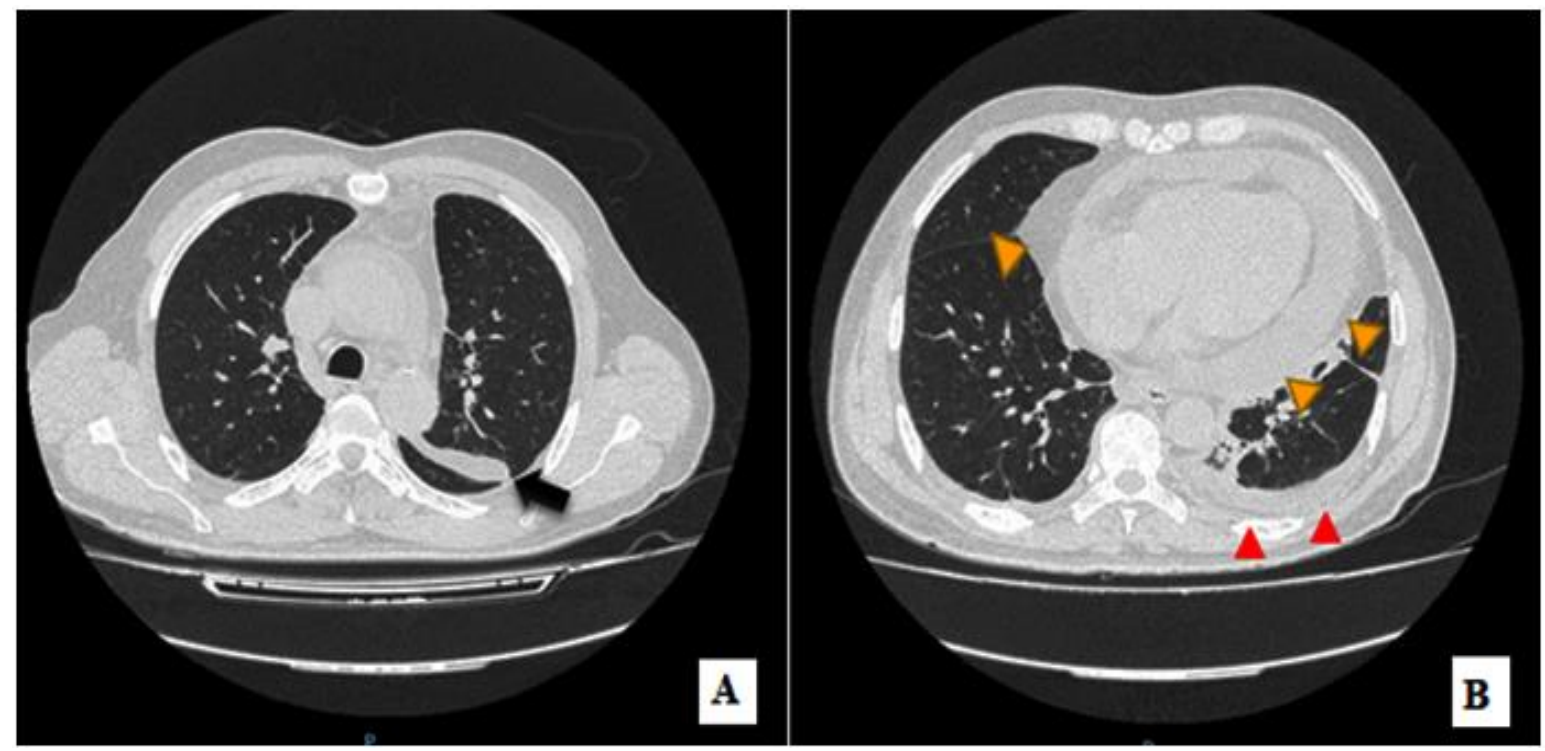

Figura 3. Tomografía de Tórax, A y B: Cortes axiales en ventana para pulmón. Se observa líquido intracisural izquierdo con atelectasia pasiva adyacente (flecha negra), derrame pleural ipsilateral (puntas de flecha roja), y derrame pericárdico con densidad de tejidos blandos (puntas de flecha amarilla).

Tabla 1. Características citoquímicas del líquido pericárdico.

\begin{tabular}{|l|l|l|}
\hline Característica & $\begin{array}{l}\text { Parámetros de } \\
\text { normalidad }\end{array}$ & $\begin{array}{l}\text { Hallazgo } \\
\text { en el } \\
\text { paciente }\end{array}$ \\
\hline Color & Claro & Amarillo \\
\hline Claridad & Claro & Turbio \\
\hline Glucosa $(\mathrm{mg} / \mathrm{dL})$ & $\begin{array}{l}\text { Paralela a los } \\
\text { valores séricos }\end{array}$ & 29,10 \\
\hline Proteína $(\mathrm{g} / \mathrm{dL})$ & No establecido & 4,95 \\
\hline $\begin{array}{l}\text { Células } \\
\text { (numero/mm }{ }^{3} \text { ) }\end{array}$ & $<300$ & 1008 \\
\hline$\%$ Neutrófilos & 0 & $20 \%$ \\
\hline$\%$ Linfocitos & 0 & $76 \%$ \\
\hline Monocitos & 0 & - \\
\hline
\end{tabular}

Con base en los hallazgos de la biopsia pericárdica y el estudio de ADA, se estableció diagnóstico definitivo de pericarditis aguda por Mycobacterium tuberculosis, descartando las demás etiologías posibles (Tabla 2), y se realizó enzimoinmunoanálisis de adsorción (ELISA) para virus de inmunodeficiencia humana (VIH), el cual fue negativo. Considerando su condición clínica, el paciente fue trasladado a la unidad de cuidados intensivos donde presentó una adecuada recuperación posquirúrgica, lo que permitió el retiro del soporte ventilatorio y el tubo de drenaje mediastinal sin complicaciones. Se trasladó a cuidados generales con un hemograma de control que evidenció disminución de la leucocitosis (Leucocitos: $11.800 / \mathrm{mm}^{3}$, con neutrofilia del $74 \%$ ) y de los marcadores inflamatorios PCR: 10,64 mg/Lt. Se inició manejo con prednisolona tabletas de $50 \mathrm{mg}$ cada 24 horas; se realizó ecocardiograma de control que reportó derrame pericárdico escaso sin signos de aumento de la presión intrapericárdica. Debido a la adecuada evolución, el paciente fue dado de alta a los 20 días de estancia hospitalaria con esquema de manejo antituberculoso ambulatorio, presentando resolución total de los síntomas durante el seguimiento posterior. 
Tabla 2. Diagnóstico diferencial del derrame pericárdico.

\begin{tabular}{|c|c|}
\hline Origen inflamatorio & Origen no inflamatorio \\
\hline 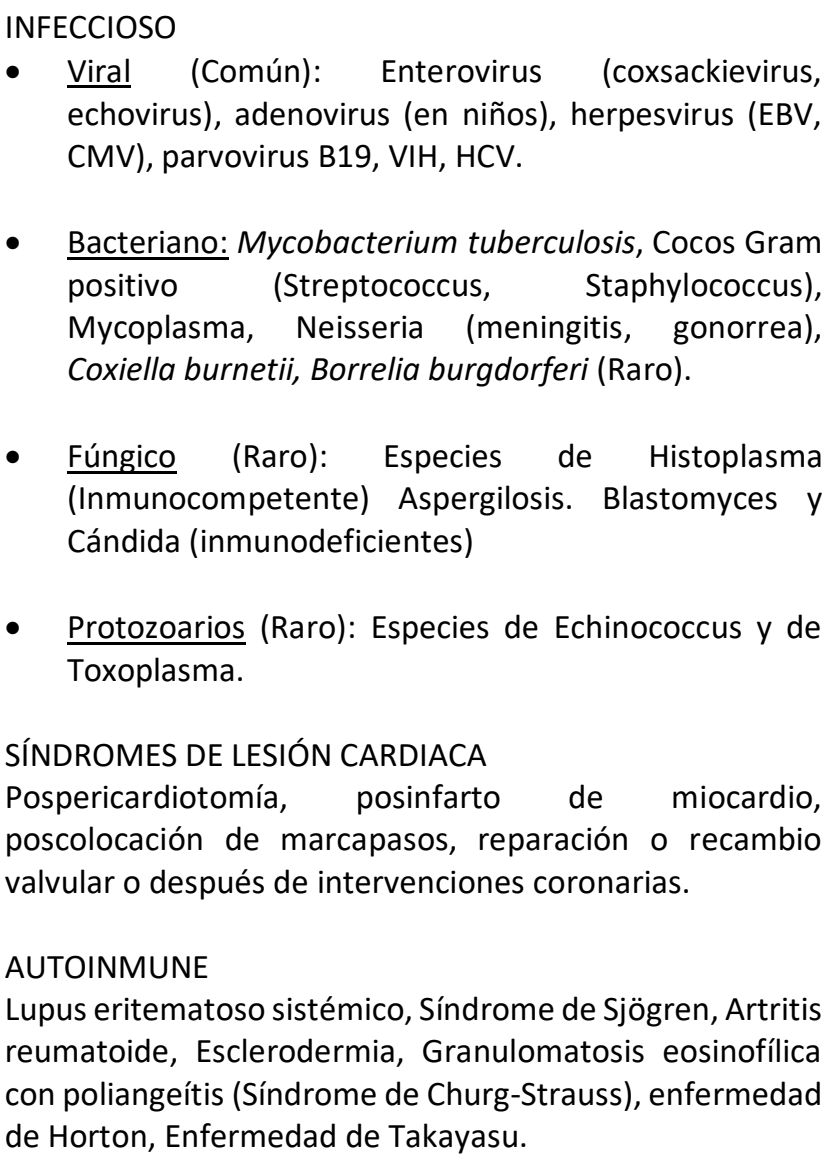 & $\begin{array}{l}\text { NEOPLÁSICO } \\
\text { Tumores primarios (mesotelioma, sarcoma) y secundarios } \\
\text { (cáncer de pulmón y mama, linfoma) } \\
\text { METABÓLICO } \\
\text { Hipotiroidismo (coma mixedematoso), hipertiroidismo, } \\
\text { anorexia nerviosa, deficiencia proteica severa. } \\
\text { TRAUMÁTICO } \\
\text { Lesión pericárdica directa/indirecta iatrogénica (lesión } \\
\text { penetrante o contundente de la pared torácica y disección } \\
\text { aórtica). } \\
\text { DRENAJE LINFÁTICO REDUCIDO } \\
\text { Insuficiencia cardíaca congestiva, cirrosis, síndrome } \\
\text { nefrótico. } \\
\text { IATROGÉNICO } \\
\text { Radioterapia, medicamentos anticoagulantes. }\end{array}$ \\
\hline
\end{tabular}

${ }^{*} \mathrm{CMV}=$ Cytomegalovirus; EBV = Epstein Barr virus; HCV = Hepatitis C virus; VIH: Virus de inmunodeficiencia humana.

\section{DISCUSIÓN}

La TB continúa siendo una emergencia de salud global. Desde 1993 cuando la OMS así la declaró, no se ha logrado una reducción significativa de su incidencia $y$, según el informe mundial sobre la tuberculosis del 2019, sigue siendo la primera causa de muerte por un agente infeccioso único, superando incluso al $\mathrm{VIH}^{9}$.

Esta enfermedad puede ser adquirida por inoculación directa (contacto directo con tejido contaminado), por ingestión de alimentos contaminados o por inhalación de unidades bacilares (forma más frecuente) $)^{3,9}$. Aunque esta patología afecta principalmente a los pulmones, puede extenderse a otros órganos y se disemina en los tejidos con mayor tensión tisular de oxígeno, por ejemplo, riñones, hígado, glándulas suprarrenales y a través del plexo venoso de Batson hacia las vertebras ${ }^{10}$. La infección se extiende hacia el pericardio, principalmente por diseminación linfática desde los ganglios pretraqueales y mediastinales, o por vecindad desde tejidos adyacentes comprometidos como pulmón o pleura $^{1,2,6}$.

La pericarditis tuberculosa se caracteriza por un cuadro clínico con síntomas que varían en intensidad, dependiendo del tiempo de instauración, puede ser aguda $(<6$ semanas) 0 crónica ( $>6$ semanas); y según la cantidad de líquido acumulado puede desarrollar tres formas de presentación clínica: derrame pericárdico, 
pericarditis constrictiva y un patrón mixto ${ }^{11}$. Es común que se presente con tos, taquicardia, fiebre, pérdida de peso, dolor torácico de localización precordial retroesternal, pudiéndose irradiar a cuello, espalda, hombro o región supraclavicular, que suele empeorar con la inspiración, el decúbito y la tos. El paciente también puede presentar disnea, pulso paradójico (definido como la caída de $>10$ $\mathrm{mmHg}$ de la presión arterial sistólica durante la inspiración), edema de miembros inferiores y hepatomegalia ${ }^{1,2,10}$. Al examen físico el signo característico de pericarditis aguda es el roce pericárdico encontrado en un $60-85 \%$ de los casos $^{16,17}$. En el caso aquí descrito, el paciente refirió fiebre subjetiva, presentaba disnea y dolor torácico pleurítico que empeoraba a la palpación en hemitórax izquierdo, y a la inspección presentaba ingurgitación yugular. Se han establecido una serie de criterios de confirmación diagnóstica. Basta con la presencia de uno para hacer el diagnóstico. Estos se describen en detalle en la Tabla $3^{12}$.

Una revisión de los métodos diagnósticos directos demuestra que la tinción clásica de Ziehl-Neelsen es poco fiable, con positividad solo hasta en el $42 \%$ de los casos. Lo mismo sucede con los cultivos de líquido pericárdico donde solo entre el $53-75 \%$ son positivos $^{6,13,14}$. Por otro lado, aunque la PCR para ADN o ARN de $M$. tuberculosis en tejido pericárdico es otra prueba de certeza diagnóstica, tiene un alto costo y baja disponibilidad, por lo que se recomienda solo en casos en los que no se ha conseguido llegar al diagnóstico por otros medios ${ }^{13}$. La biopsia pericárdica también representa un método diagnóstico eficiente, además de ser un procedimiento menor que puede ser practicado por el cirujano bajo anestesia local o general. Esta tiene una sensibilidad diagnóstica del $87 \%$ para la pericarditis tuberculosa $a^{6,15-17}$.

Dentro de los métodos indirectos para el diagnóstico se cuenta con el análisis bioquímico del líquido pericárdico, el cual tiene un aspecto serohemático en el $80 \%$ de los casos, con predominio celular linfocítico y mononuclear de tipo exudativo y alto contenido de proteínas, similar al reportado en el caso aquí presentado ${ }^{6}$. adicionalmente se puede emplear la determinación de niveles de interferón gamma (INF-g), en los que un valor de $200 \mathrm{mg} / \mathrm{dL}$ o más alcanza una sensibilidad de $92 \%$ y especificidad del $100 \%{ }^{18}$. Además, hay diversos estudios que evalúan la detección de lisozima pericárdica con un punto de corte de $6,5 \mathrm{~g} / \mathrm{dL}$ como parámetro diagnóstico, logrando una sensibilidad y especificidad de 100 y $91,17 \%$ respectivamente ${ }^{6}$.

La $A D A$ es una enzima presente en linfocitos y células mieloides. Esta se eleva en derrames de origen inflamatorio (pleurales, pericárdicos y articulares) causados por infecciones bacterianas, inflamación granulomatosa, malignidad y enfermedades autoinmunes ${ }^{19}$. Los valores de ADA en derrames de predominio linfocítico se caracterizan por tener niveles típicamente más elevados cuando la etiología es tuberculosa. En estos casos, el umbral para un resultado que favorezca el diagnóstico de tuberculosis es un valor superior a $40 \mathrm{UI} / \mathrm{L}$, el cual tiene una sensibilidad de $87-93 \%$ y especificidad de $89-97 \%$ para el diagnóstico ${ }^{20}$, valores que fueron encontrados en el presente caso. En países donde la tuberculosis es endémica, un ADA mayor a $40 \mathrm{UI} / \mathrm{L}$ en un derrame de predominio linfocítico indica una probabilidad del $96 \%$, mientras que niveles iguales o menores de $40 \mathrm{UI} / \mathrm{L}$ tienen una probabilidad del $19 \%$. A pesar de esto, se recomienda empezar tratamiento antituberculoso en pacientes con alta probabilidad, aun con valores de ADA menores a $40 \mathrm{UI} / \mathrm{L}, \mathrm{y}$ confirmar el diagnóstico con paraclínicos adicionales, definiendo el mantenimiento del tratamiento iniciado 7 .

En conclusión, la pericarditis tuberculosa, aunque es una complicación poco frecuente, implica un alto riesgo de morbilidad y mortalidad para el paciente. Además, representa un reto diagnóstico por su sintomatología clínica inespecífica, por lo que el profesional médico debe hacer uso de todas las herramientas disponibles para decidir el inicio de un tratamiento oportuno. 
Tabla 3. Parámetros diagnósticos confirmatorios de pericarditis tuberculosa.

Estudio de líquido pericárdico

1. Cultivo positivo para bacilo de Koch.

2. Hallazgo de bacilo de Koch en el extendido directo.

3. Adenosina Desaminasa (ADA) pericárdica mayor de $50 \mathrm{UI} / \mathrm{L}$.

Estudio de biopsia de tejido pericárdico

1. Cultivo positivo para bacilo de Koch.

2. Granuloma con necrosis caseosa.

3. Presencia de células gigantes multinucleadas tipo Langhas.

4. Presentación de Bacilos ácido-alcohol resistente en la muestra.

\section{DECLARACIÓN SOBRE CONFLICTOS DE INTERESES}

Declaramos no tener ningún conflicto de intereses.

\section{CONTRIBUCIÓN DE LOS AUTORES}

Primer autor: redacción

Segundo autor: trabajo de campo.

Tercer autor: trabajo de campo.

Cuarto autor: redacción y asesor de documentación.

\section{REFERENCIAS BIBLIOGRÁFICAS}

1. Trautner BW, Darouiche RO. Tuberculous Pericarditis: Optimal Diagnosis and Management. Clinical Infectious Diseases. 2001; 33(7): 954-961. Doi: https://doi.org/10.1086/322621

2. Khandaker MH, Espinosa RE, Nishimura RA, Sinak L, Hayes SN, Melduni RM, et al. (2010). Pericardial Disease: Diagnosis and Management. Mayo Clinic Proceedings. 2010; 85(6): 572-593. Doi: https://doi.org/10.4065/mcp.2010.0046

3. Murray JF. A Century of Tuberculosis. American Journal of Respiratory and Critical Care Medicine.
2004; 169(11):1181-1186. Doi: https://doi.org/10.1164/rccm.200402-140oe

4. Kekre NS, Sundaram M, Adhikary SD, John GT. Tuberculosis in renal transplant recipients. Indian Journal of Urology. 2008; 24(3), 396. Doi: https://doi.org/10.4103/0970-1591.42625

5. Raviglione MC. Mycobacterial disease, En: Harrison's Principles of Internal Medicine. EEUU: New York, 19th Editorial, McGraw-Hill Education; 2015. p.1111.

6. Echeverri D, Matta L. Pericarditis tuberculosa: reporte de caso. Biomédica. 2014; 34(4): 528-534. Doi: https://doi.org/10.7705/biomedica.v34i4.2064

7. Chau E, Sarkarati M, Spellberg B. Adenosine Deaminase Diagnostic Testing in Pericardial Fluid. JAMA. 2019; 322(2):163. Doi: https://doi.org/10.1001/jama.2019.7535

8. Imazio M, Gaita F, LeWinter M. Evaluation and Treatment of Pericarditis. JAMA. 2015; 314(14), 1498-1506.

Doi: 
9. Organización Mundial de la Salud. Informe mundial sobre la tuberculosis 2019. Washington DC: OMS; 2019.

10. Aguilar JA, Summerson C, Montoya GE, González GG, Corvala JA, Godínez RJ, et al. Pericarditis tuberculosa: Experiencia de 10 años. Archivos de Cardiología de México. [revista en la Internet]. 2007 [citado 2020 Mayo 14]; 77:209-216. Disponible en: http://www.scielo.org.mx/pdf/acm/v77n3/v77n3a 5.pdf

11. Ferrari SM, Garcia HL, Durón LP. Pericarditis tuberculosa: informe de caso. Rev. méd. Hondur. [revista en la Internet]. 2010 [citado 2020 Mayo 14]; 78(1): 25-28. Disponible en: http://www.bvs.hn/RMH/pdf/2010/pdf/Vol78-12010-9.pdf

12. Castañeda SE, Mejía JM. Valor del líquido pericárdico con relación a la biopsia pericárdica en el diagnóstico de pericarditis efusiva tuberculosa. Revista Médica Heredianat. [revista en la Internet]. 2000 [citado 2020 Mayo 14]; 11(4): 122$129 . \quad$ Disponible en: http://www.scielo.org.pe/pdf/rmh/v11n4/v11n4ao 2.pdf

13. Cegielski JP, Devlin BH, Morris AJ, Kitinya JN, Pulipaka UP, Lema LE, et al. Comparison of PCR, culture, and histopathology for diagnosis of tuberculous pericarditis. Journal of Clinical Microbiology. [revista en la Internet]. 1997 [citado 2020 Mayo 14]; 35: 3254-3257. Disponible en: https://www.ncbi.nlm.nih.gov/pmc/articles/PMC23 0157/pdf/353254.pdf

14. Amin-Beidokhty A, Norouzi Z, Amiri A, Almasian $M$, Azadi A, Kheirollahi AR. Pericardial tuberculosis with an emphasis on empiric therapy in endemic areas for tuberculosis (a case report). International Journal of Mycobacteriology. 2016; 5(3), 360-365. Doi: https://doi.org/10.1016/j.ijmyco.2016.08.006

15. Reuter H, Burgess L, Van Vuuren W, Doubell A. Diagnosing tuberculous pericarditis. QJM, an International Journal of Medicine. 2006; 99(12): 827-839.

Doi:
16. Maltezou HC, Spyridis P, Kafetzis DA. Extrapulmonary tuberculosis in children. Archives of Disease in Childhood. 2000; 83(4): 342-346. Doi: https://doi.org/10.1136/adc.83.4.342

17. Clavería RC, Vergara GL, Negrón MS, López LC, Zelada PP, Carrasco OJ. Derrame Pericárdico, Enfrentamiento Clínico. Revista Chilena de Pediatría. [revista en la Internet]. 2009 [citado 2020 Mayo 14]; 80(3): 267-273. Disponible en: https://www.revistachilenadepediatria.cl/index.ph $\mathrm{p} / \mathrm{rchped} /$ article/view/2576

18. Yusuf SW, Hassan SA, Mouhayar E, Negi SI, Banchs J, O'Gara PT. Pericardial disease: a clinical review. Expert Review of Cardiovascular Therapy. 2016; 14(4): 525-539. Doi: https://doi.org/10.1586/14779072.2016.1134317

19. Arroyo M, Soberman JE. Adenosine Deaminase in the Diagnosis of Tuberculous Pericardial Effusion. The American Journal of the Medical Sciences. 2008; 335(3): 227-229. Doi: https://doi.org/10.1097/maj.0b013e3180cab71a

20. Koh KK, Kim EJ, Cho CH, Choi MJ, Cho SK, Kim SS, et al. Adenosine deaminase and carcinoembryonic antigen in pericardial effusion diagnosis, especially in suspected tuberculous pericarditis. Circulation. 1994; 89(6): 2728-2735. Doi: https://doi.org/10.1161/01.cir.89.6.2728 https://doi.org/10.1093/qjmed/hcl123 